\title{
Bacteriemias por Streptococcus gallolyticus (ex S. bovis) y su relación con patología colónica o hepatobiliar y endocarditis
}

\author{
Cristina Amado, M. José Hidalgo, Cecilia Sedano, Allan Hebel, Lorena Porte, \\ Stephanie Braun, Jeannette Dabanch y Alberto Fica
}

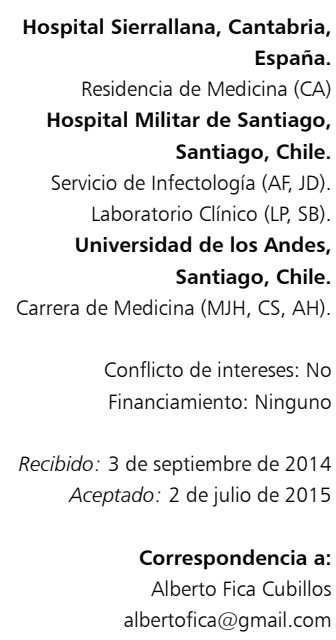

Alberto Fica Cubillos

\section{Streptococcus gallolyticus (ex S. bovis) bacteremia and its relationship with colonic or hepatobiliary disease and endocarditis}

Background: Bacteremia due to Streptococcus bovis (now S. gallolyticus) has been traditionally associated to colon or hepatobiliar disease and endocarditis but there is no information on this matter in Chile. Aims: To describe clinical features of adult patients suffering bacteremia by $S$. bovis/S. gallolyticus, identify the source of the bacteremia and the frequency of endocarditis. Methods: Retrospective-descriptive study using laboratory records. Results: Between January 2003 and August 2014, 23 S. bovis/S. gallolyticus bacteremic events were identified among 22 patients. Mean age was 72.7 years (range 46-96). Co-morbidities were frequent (9.1 to 47.6\%). The primary source of bacteremia was intestinal in $52.2 \%$; hepatobiliar in $17.4 \%$ and in $34.8 \%$ it was not elucidated. Six patients had infective endocarditis $(26.1 \%)$ and one patient had espondylodiscitis $(4.3 \%)$. S. bovis represented $39.1 \%$ of isolates (all until 2008), S. gallolyticus subsp pasteurianus $39.1 \%$ and, S. gallolyticus subsp infantarius and $S$. gallolyticus subsp gallolyticus $8.7 \%$ each one, respectively. Association studies between the bacteremic source or endocarditis with specific $S$. gallolyticus subspecies were limited by the small number of isolates. Seven patients $(30.4 \%)$ underwent surgical interventions. In-hospital mortality reached $21.7 \%(\mathrm{n}=5)$. Conclusions: Although infrequent, bacteremic events by $S$. gallolyticus/S. bovis have increased in-hospital mortality, require surgical intervention and affect older patients with co-morbidities. Near two-thirds suffer from colonic or hepatobiliary disease that act as the primary source of bacteremia. In addition, near one fourth is affected by infective endocarditis. Detection of $S$. gallolyticus/S. bovis in blood cultures prompts a thorough clinical evaluation in order to clarify the source of the bloodstream infection and the presence of complications.

Key words: Streptococcus bovis; bacteremia; infective endocarditis; sigmoid colon disease; biliary tract disease, adult.

Palabras clave: Streptococcus bovis; bacteremia; endocarditis infecciosa; enfermedad colon sigmoides; enfermedad del tracto biliar, adulto.

\section{Antecedentes}

\section{L}

os cuadros de bacteriemia por Streptococcus bovis han sido tradicionalmente asociados a endocarditis y/o patología colónica o hepatobiliar ${ }^{1-3}$. Los cambios taxonómicos han definido de mejor manera estas relaciones $^{4}$, estableciendo que el biotipo I, ahora denominado Streptococcus gallolyticus subsp gallolyticus es el grupo que mayormente se asocia con endocarditis y cáncer de colon. En contraste, el antiguo biotipo II tiene más relación con bacteriemias de origen hepatobiliar ${ }^{5}$, grupo constituido ahora por S. gallolyticus subsp. infantarius y S. gallolyticus subsp. pasteurianus. Las asociaciones comentadas para el biotipo I son relevantes ya que deberían determinar una búsqueda activa de endocarditis y patología colónica cada vez que se identifica este grupo en los hemocultivos. Sin embargo, y debido a que las fronteras no son absolutas, el biotipo II también tiene cierta relación con endocarditis y patología benigna del colon. Explorar las asociaciones entre diferentes biotipos de $S$. bovis o en forma más precisa, subespecies de $S$. gallolitycus con la existencia de endocarditis, patología benigna o maligna de colon o hepatobiliar es de gran importancia para orientar a los equipos de salud en la búsqueda de estos problemas. No se conoce de estudios similares en Chile que hayan abordado esta situación. Los objetivos de este trabajo fueron describir los aspectos clínicos de pacientes adultos afectados por bacteriemias debidas a diferentes subespecies de $S$. gallolyticus, identificar la fuente de la bacteriemia, las subespecies específicas involucradas, la frecuencia de endocarditis e intentar establecer una asociación entre subespecies y patología colónica, hepatobiliar y endocarditis.

\section{Pacientes y Métodos}

Diseño de tipo retrospectivo, descriptivo, tipo serie clínica. Para la pesquisa de casos se utilizó el registro de casos disponibles en el Laboratorio de Microbiología del Hospital Militar. Se incluyeron casos con hemocultivos positivos a las subespecies o biotipos comentadas o con amplificación molecular positiva desde válvula cardíaca.

De cada caso se extrajo información demográfica, antecedentes mórbidos generales y de relevancia para este tipo de infecciones (adulto mayor, diabetes mellitus, 
inmunosupresión, cáncer, cardiopatía valvular de base), manifestaciones clínicas parámetros del examen físico, variables de laboratorio, estudio de imágenes efectuados incluyendo ecocardiografía, estudios digestivos bajos, biopsias y sus resultados, unidad de hospitalización de mayor complejidad, tratamiento antimicrobiano y su oportunidad, susceptibilidad antimicrobiana, evolución y condición al alta. Las cepas fueron catalogadas como Streptococcus grupo bovis hasta mediados del 2009 y desde ese época como $S$. gallolyticus subsp gallolyticus (ex $S$. bovis biotipo I), $S$. gallolyticus subsp pasteurianus (ex S. bovis biotipo II/2) o S. gallolyticus subsp infantarius (ex S. bovis biotipo II/1) por la incorporación en el hospital de un sistema automatizado de identificación Vitek que permitió tal identificación.

\section{Definiciones}

La patología colónica se definió en este trabajo según la presencia de tumor, pólipos, engrosamiento parietal del colon o el hallazgo de una gran cantidad de deposiciones en el lumen intestinal, fuese por tomografía o colonoscopia. Se consideró que el paciente no fue evaluado si no se efectuó una colonoscopia como parte del estudio. Por su parte, la presencia de patología hepatobiliar se definió por el hallazgo de un cuadro agudo ya fuese como absceso hepático o patología biliar aguda. Los casos de endocarditis fueron identificados por los criterios de Duke modificados.

La información se presenta en forma descriptiva y los estudios de asociación se hicieron mediante pruebas no paramétricas por el reducido número de casos obtenidos ya fuera con la prueba bilateral de Fisher para variables categóricas o la prueba de Mann-Whitney para comparar variables continuas entre diferentes grupos. Para el análisis de subgrupos se utilizó la mediana como medida de tendencia central y el rango interquartil (RIQ) 25-75\% como medida de dispersión. El trabajo fue aprobado por el Comité de Ética del Hospital Militar.

\section{Resultados}

Entre enero de 2003 y agosto de 2014 se identificaron 23 eventos de bacteriemia por $S$. bovis/S. gallolyticus en 22 pacientes. Un paciente tuvo dos eventos separados por dos años y ambos casos con endocarditis. La frecuencia anual de casos osciló entre uno y cinco por año sin observarse una tendencia al aumento. No hubo un predominio de género y la edad promedio fue de 72,7 años (rango 46-96). Cerca de la mitad de los pacientes tenía $\geq 75$ años y la prevalencia de co-morbilidades fue elevada (Tabla 1).

Los pacientes se presentaron con un promedio sintomático de nueve días antes del ingreso y predominantemente con fiebre. Otras manifestaciones incluyeron compromiso de conciencia y, con menor frecuencia, dolor abdominal e hipotensión arterial, entre otras (Tabla 2). Se puede apreciar también que la diversidad sintomática fue importante. En el examen físico destacaron la presencia de desnutrición (n: 6; 26,1\%), soplos cardiacos (n: 6; $26,1 \%$, todos de tipo sistólico) y distensión abdominal (n: 5; 21,7\%). Los soplos estuvieron significativamente asociados a la existencia de endocarditis infecciosa en el subgrupo con evaluación ecocardiográfica disponible. Así, cinco de seis pacientes con soplos tuvieron endocarditis versus uno de 11 sin este hallazgo (Odds ratio 50; IC95 2,5 a 977; $p<0,01$ por prueba bilateral de Fisher). Por otra parte, no se observó una asociación entre cambio del hábito intestinal y patología de colon.

\section{Hallazgos de laboratorio}

El 68,2\% (n: 15) de los pacientes presentó cifras de hemoglobina en rango de anemia (hemoglobina $<12 \mathrm{~g} /$ $\mathrm{dL}$ ), $47,8 \%$ leucocitosis (recuento $\geq 12.000 / \mu \mathrm{L}$ ) pero sólo uno tuvo VHS $>100 \mathrm{~mm} / \mathrm{h}(4,3 \%)$. Además, $50 \%$ tenía hipoalbuminemia $<3,5 \mathrm{~g} / \mathrm{dL}$ y $61,1 \%$ cifras de LDH plasmática elevada $(>250 \mathrm{U} / \mathrm{L})$. Los valores de transaminasas

Tabla 1. Características demográficas y co-morbilidades en 23 eventos de bacteriemia por S. bovis/S. gallolyticus, HMS 2003-2014

\begin{tabular}{|lll|}
\hline Condición & $\mathbf{n} / \mathbf{N}$ & Resultado \\
\hline Edad valor promedio (rango) en años & 22 & $72,7(46-96)$ \\
$\quad$ Edad $\geq 60$ & $18 / 23$ & $78,3 \%$ \\
$\quad$ Edad $\geq 75$ & $12 / 23$ & $52,2 \%$ \\
\hline Sexo masculino & $12 / 22$ & $54,5 \%$ \\
\hline Diabetes mellitus tipo 2* & $10 / 21$ & $47,6 \%$ \\
Cardiopatía* & $7 / 21$ & $33,3 \%$ \\
Cáncer sólido & $5 / 22$ & $22,7 \%$ \\
Enfermedad renal crónica* & $4 / 22$ & $18,2 \%$ \\
\hline Daño hepático crónico & $4 / 22$ & $18,2 \%$ \\
Diálisis & $2 / 22$ & $9,1 \%$ \\
\hline Linfoma-leucemia & $2 / 22$ & $9,1 \%$ \\
\hline EPOC u otra enfermedad pulmonar crónica* & $1 / 21$ & $4,8 \%$ \\
\hline Corticoides en dosis inmunosupresoras & $1 / 22$ & $4,5 \%$ \\
\hline
\end{tabular}

*En un paciente con daño cognitivo demencial no se contó con información sobre diabetes mellitus, EPOC o cardiopatía.

Tabla 2. Presentación clínica en 23 eventos de bacteriemia por S. bovis/S. gallolyticus, HMS 2003-2014

\begin{tabular}{|c|c|c|}
\hline Manifestación clínica & $n / N$ & Resultado \\
\hline Fiebre & $17 / 23$ & $73,9 \%$ \\
\hline Compromiso de conciencia & $9 / 23$ & $39,1 \%$ \\
\hline Dolor abdominal & $5 / 23$ & $21,7 \%$ \\
\hline Hipotensión arterial (sistólica < 90 o diastólica < 60 mmHg) & $5 / 23$ & $21,7 \%$ \\
\hline Insuficiencia respiratoria $\left(\mathrm{SO}_{2}<90 \%\right.$ con aire ambiental) & $4 / 23$ & $17,4 \%$ \\
\hline $\begin{array}{l}\text { Cambio de hábito intestinal } \\
\text { Constipación reciente o en aumento } \\
\text { Diarrea reciente o intermitente }\end{array}$ & $\begin{array}{l}6 / 23 \\
2 / 23 \\
4 / 23\end{array}$ & $\begin{array}{r}26,1 \% \\
8,7 \% \\
17,4 \%\end{array}$ \\
\hline Déficit neurológico focal & $3 / 23$ & $13,0 \%$ \\
\hline Insuficiencia cardíaca & $3 / 23$ & $13,0 \%$ \\
\hline Baja de peso & $3 / 23$ & $13,0 \%$ \\
\hline Diaforesis & $2 / 23$ & $8,7 \%$ \\
\hline Hemorragia digestiva baja & $1 / 23$ & $4,3 \%$ \\
\hline Duración de los síntomas hasta el ingreso promedio (rango) & 23 & $9,3(0-30)$ \\
\hline n consultas hasta el ingreso promedio (rango) & 22 & $0,5(0-4)$ \\
\hline
\end{tabular}


glutámico-oxaloacética (SGOT) y glutámico-pirúvica (SGPT) fueron significativamente superiores en el grupo de los cuatro pacientes con patología hepatobiliar respecto a los 15 pacientes en los que ésta fue excluida (mediana de SGOT de 549 UI/L [RIQ 289-618] y de SGPT de 201 UI/L [RIQ 197-408] versus 34 [RIQ 23-37] y 20 UI/L [RIQ 10-26], respectivamente; $\mathrm{p}<0,01$ por prueba de Mann-Whitney). A su vez, los valores de creatininemia y albuminemia estuvieron significativamente más elevados en el grupo portador de patología colónica respecto al grupo sin esta condición o no evaluado para ella. Para creatininemia medianas de $1,8 \mathrm{mg} / \mathrm{dL}$ (RIQ 0,9-2,4) versus $1 \mathrm{mg} / \mathrm{dL}$ (RIQ 0,6-1,2) (p<0,05 por prueba de Mann-Whitney) y en el caso de la albuminemia, medianas de 3,85 g/dL (RIQ 3,55-4,02) versus 3,2 g/dL (RIQ 2,7$3,4)(\mathrm{p}<0,05$ por prueba de Mann-Whitney).

Foco primario por patología colónica, hepatobiliar y frecuencia de endocarditis y espondilodiscitis

El foco primario de la bacteriemia fue intestinal en aproximadamente la mitad de los pacientes tal como queda evidenciado por anormalidades en los estudios por

Tabla 3. Endocarditis, espondilodiscitis, patología colónica y hepatobiliar en 23 eventos de bacteriemia por S. bovis/S. gallolyticus, HMS 2003-2014

\begin{tabular}{lrr} 
Parámetro & $\mathbf{n}$ & Resultado \\
Patología colónica & 1 & \\
Cáncer de colon & 6 & $26,1 \%$ \\
Pólipo benigno & 2 & $8,7 \%$ \\
Impacto fecal & 2 & $8,7 \%$ \\
Engrosamiento parietal (por tomografía) & 1 & $4,3 \%$ \\
Obstrucción intestinal & 12 & $52,2 \%$ \\
Total con lesiones en endoscopia o tomografía computada & 15 & $65,2 \%$ \\
Sin evaluación endoscópica & & \\
Patología hepatobiliar & 1 & $4,3 \%$ \\
Colangitis & 1 & $4,3 \%$ \\
Colecistitis aguda & 1 & $4,3 \%$ \\
Pancreatitis aguda biliar & 1 & $4,3 \%$ \\
Absceso hepático & $17,4 \%$ \\
Total con patología hepatobiliar aguda & 4 & $17,4 \%$ \\
Sin evaluación por imágenes & 4 & $34,8 \%$ \\
Sin foco de bacteriemia aclarado & 8 & $26,1 \%$ \\
Endocarditis infecciosa presente & 6 & $26,1 \%$ \\
Casos sin evaluación & $6,3 \%$ \\
\hline Espondilodiscitis & 6 & \\
\hline
\end{tabular}

Tabla 4. Distribución de especies y subespecies en 23 eventos de bacteriemia por S. bovis/S. gallolyticus, HMS 2003-2014

\begin{tabular}{lrr} 
Grupo bacteriano & $\mathbf{n}$ & Resultado \\
Streptococcus grupo bovis* & 9 & $39,1 \%$ \\
Streptococcus grupo bovis por secuenciación molecular & 1 & $4,3 \%$ \\
Streptococcus gallolyticus subsp. gallolyticus (Ex S. bovis biotipo I) & 2 & $8,7 \%$ \\
Streptococcus gallolyticus subsp. pasteurianus (Ex S. bovis biotipo II/2) & 9 & $39,1 \%$ \\
Streptococcus gallolyticus subsp. infantarius (Ex S. bovis biotipo II/1) & 2 & $8,7 \%$ \\
Total & 23 & $100,0 \%$ \\
\hline *Sin especificación, cepas estudiadas antes del 2009. & & \\
\hline
\end{tabular}

colonoscopia o tomografía computada (Tabla 3). Las alteraciones pesquisadas incluyeron un caso de cáncer de colon, pólipos, engrosamiento parietal detectado en la tomografía (sin colonoscopia), obstrucción intestinal y dos casos de impactación fecal en el colon. Cerca de dos tercios de la serie no tuvo una evaluación por endoscopía y este hecho estuvo simétricamente distribuido en pacientes de mayor o menor edad (datos no mostrados) y en al menos cinco casos $(21,7 \%)$ este examen fue rechazado, postergado o desestimado por el paciente, familiares o el equipo médico.

La patología hepatobiliar aguda fue la segunda causa de bacteriemia identificada pero con una frecuencia menor que la colónica e incluyó principalmente patología relacionada a la vía biliar (Tabla 3 ).

En ocho casos no se determinó el origen de la bacteriemia $(34,8 \%)$, ya fuese por estudio incompleto o estudio abdominal negativo. En este estudio, seis pacientes presentaron endocarditis infecciosa $(26,1 \%)$ y uno espondilodiscitis $(4,3 \%)$ como complicación de la bacteriemia.

\section{Distribución por especie y subespecies}

La distribución por especies y subespecies se indica en la Tabla 4. Hasta el año 2008, sólo era posible la identificación por $S$. grupo bovis lo que explica la distribución observada. Una mayor precisión a nivel de subespecie fue posible desde el año 2009. En esta etapa, se observó un claro predominio de $S$. gallolyticus subsp. pasteurianus (Ex S. bovis biotipoII/2). Un caso de la serie fue identificado desde tejido valvular cardíaco mediante secuenciación del ARN16S. En 18 de 22 casos $(81,8 \%)$ con hemocultivos positivos, hubo crecimiento monomicrobiano y en el resto (n: $4 ; 18,2 \%$ ) polimicrobiano. Esta característica no estuvo asociada significativamente a un origen colónico o biliar.

\section{Estudio de asociación entre grupos microbianos y endocarditis, patología colónica o hepatobiliar}

Los estudios de asociación estuvieron limitados por el bajo número de aislados con identificación a nivel de subespecie (n: 13). En el sub grupo con información ecocardiográfica y de subespecie se observó una tendencia entre bacteriemia por $S$. gallolyticus subsp. pasteurianus y ausencia de endocarditis (p: 0,3). De los seis casos de endocarditis, cinco estuvieron asociados a $S$. grupo bovis sin tipificación y uno a $S$. gallolytcus subsp gallolyticus.

La distribución de los diferentes grupos bacterianos y patología de colon se indica en la Tabla 5. Se puede apreciar la amplitud de patología asociada a la subespecie pasteurianus.

\section{Manejo y evolución}

Todos los pacientes recibieron antimicrobianos en su evolución y en todos los casos de espectro adecuado. Siete pacientes $(30,4 \%)$ debieron ser intervenidos quirúrgicamente, cirugía cardiovascular (n: 4) o abdominal (n: 3). Otros dos pacientes $(8,7 \%)$ recibieron procedimientos intervencionales consistentes en una punción percutánea para el manejo de un absceso hepático y una colangiogra- 


\begin{tabular}{|c|c|c|c|c|c|c|}
\hline Grupo & Cáncer (n) & Pólipo (n) & Impactación fecal (n) & Engrosamiento parietal (n) & Obstrucción intestinal (n) & Total \\
\hline S. bovis sin especificar & 0 & 4 & 0 & 0 & 0 & 4 \\
\hline Subsp gallolyticus & 0 & 1 & 0 & 0 & 0 & 1 \\
\hline Subsp infantarius & 0 & 0 & 0 & 0 & 1 & 1 \\
\hline Subsp pasteurianus & 1 & 1 & 2 & 2 & 0 & 6 \\
\hline
\end{tabular}

fia retrógada endoscópica con extracción de cálculos. La mayor parte de los pacientes debió ser manejado en UCI (n: $8 ; 34,8 \%$ ) o Sala Intermedia (n: 9; 39,1\%). La estadía hospitalaria promedio fue de 20,1 días (rango 1-51). La mortalidad hospitalaria fue de $21,7 \%$ (n: 5). El único factor asociado a un mayor riesgo de muerte fue la presencia de un cáncer hematológico. Dos de cinco pacientes fallecidos tenían esta condición versus ninguno de los sobrevivientes ( $\mathrm{p}<0,05$ por prueba bilateral de Fisher).

\section{Discusión}

Los resultados de este trabajo revelan que aunque infrecuentes, los eventos de bacteriemia por $S$. gallolyticus $/ S$. bovis se asocian a una morbilidad prolongada, tienen una elevada mortalidad hospitalaria, requieren con frecuencia procedimientos quirúrgicos o invasores, ocupan camas críticas o semicríticas y afectan a pacientes mayores con diferentes tipos de co-morbilidades. Además, cerca de dos tercios padecen de una patología colónica o hepatobiliar que actúa como foco primario y cerca de un cuarto presenta endocarditis infecciosa. Las características sobre comorbilidad, edad, asociación con patología hepatobiliar o colónica, endocarditis y mortalidad, son concordantes con otros reportes (Tabla comparativa en referencia 5); sin embargo, la edad promedio de nuestra serie supera otras experiencias ${ }^{5,6}$.

Aunque la existencia de patología colónica, hepatobiliar o de endocarditis, puede ser sospechada sobre las bases de la historia clínica y el examen físico, se requiere el concurso de una evaluación exhaustiva para establecer por una parte el origen de la bacteriemia y por otra, la presencia de complicaciones. Debido a los dos focos primarios documentados para bacteriemia, la evaluación del origen de ésta necesita un estudio hepatobiliar y colónico que debe contemplar una colonoscopia por la incapacidad de los estudios de imágenes para detectar patología intraluminal. Como se observó en este trabajo, todos los casos de bacteriemia por este grupo microbiano, independiente de la tipificación a nivel de subespecie, pueden asociarse a un origen biliar o colónico, por lo que esta estrategia no debe ser supeditada a la subespecie aislada. La colonoscopia no sólo permite el diagnóstico, sino que también la remoción de pólipos y en nuestra serie sólo fue efectuada tras el segundo evento de endocarditis en uno de nuestros pacientes. De esta manera debe enfatizarse el estudio colónico en todos los pacientes con bacteriemias por este grupo de microorganismos aunque el foco no sea aparente.
En nuestra serie consideramos la impactación fecal como una patología colónica. Diversos reportes señalan que el manejo de esta condición con enemas se asocia a un riesgo de sepsis grave, perforación, hospitalización o muerte ${ }^{7,8}$. Aunque los enemas no estuvieron involucrados en el manejo de nuestros pacientes, es posible que la fragilidad de ellos (edad extrema asociada a co-morbilidades) hubiera facilitado una traslocación bacteriana intestinal como los antecedentes parecen indicar. En ambos casos, no se encontró otra fuente de bacteriemia en el estudio de imágenes.

En contraste, el riesgo de endocarditis parece depender finamente de la subespecie identificada. Son varios los reportes que han establecido una estrecha asociación entre la presencia de endocarditis y el hallazgo de $S$. gallolyticus subsp gallolyticus (Ex S. bovis biotipo I) pero en menor grado con otras subespecies o biotipos ${ }^{1-3}$. De esta manera, la tendencia a una falta de asociación entre endocarditis y S. gallolyticus subsp pasteurianus, es concordante con estas descripciones en la literatura médica. Se ha establecido en algunos reportes que existe $94 \%$ de asociación entre bacteriemia por $S$. bovis del biotipo I (ahora S. gallolyticus subsp gallolyticus) y endocarditis infecciosa y por otra parte, $71 \%$ de asociación entre bacteriemia por $S$. bovis biotipo I y carcinoma del colon. En forma más reciente, un estudio casocontrol de pacientes con bacteriemia por S. gallolyticus subsp. gallolyticus sometidos a estudio endoscópico bajo y comparando los hallazgos con un grupo control sometido a colonoscopias por diferentes razones, encontró que los pacientes con bacteriemia tenían un riesgo cinco veces mayor de portar neoplasias colo-rectales. Específicamente 2,5 veces mayor para adenomas avanzados y 2,9 veces para carcinoma invasor ${ }^{9}$. En la otra vereda, las bacteriemias por aislados del biotipo II tienen una asociación $<20 \%$ con endocarditis 5 . Se ha descrito que los pacientes que padecen una patología hepatobiliar son afectados principalmente por bacteriemias debidas a $S$. gallolyticus subsp. pasteurianus (biotipo II/2) o S. infantarius (biotipo II/1) ${ }^{10}$. En línea con este comentario, dos de nuestros casos hepatobiliares con información disponible, se asociaron a $S$. gallolyticus subsp. infantarius (Ex S. bovis biotipo II/1). Otros estudios indican que cualquier subespecie tiene el mismo potencial de indicar una neoplasia maligna en el colon o recto, vía biliar e hígado, estómago o páncreas, ya que no se han podido establecer diferencias significativas a favor de una asociación en particular ${ }^{11}$. De esta manera, una bacteriemia por cualquiera de estas subespecies obliga a iniciar un estudio del foco de origen para descartar una neoplasia del tracto gastrointestinal. Se debe destacar, sin embargo, que 
las infecciones por la subespecie infantarius parecen tener un peor pronóstico que las otras variantes del grupo debido a su asociación con shock séptico y falla multiorgánica ${ }^{11}$. Al respecto, uno de nuestros casos falleció a raíz de una bacteriemia por $S$. gallolyticus subsp infantarius con shock séptico (datos no mostrados).

En nuestra serie no pudimos establecer claramente una asociación entre subespecies de S. gallolyticus y la presencia de cáncer de colon y/o endocarditis debido a la relativamente reciente incorporación de un sistema automatizado capaz de establecer esta identificación, lo que impidió asignar los casos con $S$. bovis a diferentes fuentes de origen o complicación observada. Además, un subgrupo de pacientes no contó con estudio intestinal por lo que la prevalencia de patología colónica pudiera ser mayor a la calculada y nuestros resultados deben ser tomados sólo como un mínimo.

La asociación significativa entre aumento de transaminasas y patología hepatobiliar es útil para orientar al equipo tratante sobre este foco en casos de bacteriemia por este grupo microbiano. No obstante, no pudimos interpretar adecuadamente la asociación significativa entre mayores valores de creatininemia y patología de colon presente ya que los pacientes con enfermedad renal crónica no se asociaron a esta patología en esta serie ni tampoco esta alza a la gravedad inicial del caso (datos no mostrados).

La mortalidad observada en esta serie es relevante (cerca de $20 \%$ ) y quizás superior al rango reportado en otras experiencias (10-15\%), excepto para el grupo $S$. infantarius $(30 \%)^{9-11}$. Los factores de riesgo descritos asociados a mortalidad corresponden a shock séptico y cáncer, y al menos este último fue también identificado en este trabajo ${ }^{9-11}$.

En conclusión, las bacteriemias por $\mathrm{S}$. bovis/S. gallolyticus son eventos infrecuentes de alta morbi-mortalidad siendo importante la búsqueda del foco primario, de complicaciones y donde el estudio microbiológico a nivel de subespecie permite orientar el estudio.

\section{Resumen}

Antecedentes: Los cuadros de bacteriemia por Streptococcus bovis (actualmente S. gallolyticus) han sido tradicionalmente asociados a patología colónica o hepatobiliar y endocarditis pero no se conoce de estudios en Chile que hayan abordado este tema. Objetivos: Describir aspectos clínicos de pacientes adultos afectados por bacteriemias por $S$. bovis/S. gallolyticus, identificar la fuente de la bacteriemia y la frecuencia de endocarditis. Métodos: Diseño de tipo retrospectivo, descriptivo, con el registro de casos bacteriemia. Resultados: Entre enero de 2003 y agosto de 2014 se identificaron 23 eventos de bacteriemia por $S$. bovis/S. gallolyticus en 22 pacientes. La edad promedio fue de 72,7 años (rango 46-96). La prevalencia de diferentes co-morbilidades fue elevada $(9,1$ a $47,6 \%)$. El foco primario de la bacteriemia fue intestinal en $52,2 \%$, hepatobiliar en $17,4 \%$ y, en $34,8 \%$ no se aclaró el foco. Seis pacientes presentaron endocarditis infecciosa $(26,1 \%)$ y uno espondilodiscitis (4,3\%). S. bovis representó 39,1\% de los aislados (todos hasta el 2008), S. gallolyticus subsp pasteurianus $39,1 \%, S$. gallolyticus subsp infantarius y S. gallolyticus subsp gallolyticus $8,7 \%$, respectivamente. Los estudios de asociación estuvieron limitados por el bajo número de aislados. Siete pacientes $(30,4 \%)$ debieron ser intervenidos quirúrgicamente. La mortalidad hospitalaria fue de 21,7\% (n: 5). Conclusiones: Aunque infrecuentes, los eventos de bacteriemia por $S$. gallolyticus/S. bovis tienen una elevada mortalidad hospitalaria, requieren con frecuencia procedimientos quirúrgicos y afectan a pacientes mayores con co-morbilidades. Cerca de dos tercios padecen de una patología colónica o hepatobiliar que actúa como foco primario y cerca de un cuarto presenta endocarditis infecciosa. La detección de este grupo bacteriano en los hemocultivos requiere una evaluación para establecer el origen de la bacteriemia y la presencia de complicaciones.

\section{Referencias bibliográficas}

1.- Boleij A, van Gelder M M H, Swinkels D W, Tjalsma H. Clinical importance of Streptococcus gallolyticus infection among colorectal cancer patients: Systematic review and meta-analysis. Clin Infect Dis 2011; 53: 870-8.

2.- Ruoff K L, Miller S I, Garner C V, Ferraro M J, Calderwood S B. Bacteremia with Streptococcus bovis and Streptococcus salivarius: Clinical correlates of more accurate identification of isolates. J Clin Microbiol 1989; 27: 305-8.

3.- Vaska V L, Faoagali J L. Streptococcus bovis bacteremia: identification within organism complex and association with endocarditis and colon malignancy. Pathology 2009; 41: 183-6.

4.- Spellerberg B, Brandt C. Chapter 20: Streptococcus. Versalovic J, Carroll C K, Funke G, Jorgensen J H, Landry M L, Warnock D W, editors. Manual of Clinical
Microbiology, $10^{\text {th }}$ Ed. Washington, DC: ASM Press; 2011; p. 331-49.

5.- Lee R A, Woo P C Y, To A P C, Lau S K P, Wong S S Y, Yuen K Y. Geographical difference of disease association in Streptococcus bovis bacteremia. J Med Microbiol 2003; 52: 903-8.

6.- Lazarovitch T, Shango M, Levine M, Brusovansky R, Akins R, Hayakawa K, et al. The relationship between the new taxonomy of Streptococcus bovis and its clonality to colon cancer, endocarditis, and biliary disease. Infection 2013; 41: 329-37.

7.- Niv G, Grinberg T, Dickman R, Wasserberg N, Niv Y. Perforation and mortality after cleansing enema for acute constipation are not are but are preventable. Int J Gen Med 2013; 6: 323-8.

8.- Soto A, Fica A, Dabanch J, Weitzel T. Bacteremia y sepsis grave asociada al uso de enemas. Análisis de una serie en un hospital general. Presentado en el XVI Congreso
Panamericano de Infectología, Santiago, Chile. Mayo/2013. PO 16. Disponible en: http:// www.sochinf.cl/documentos/2013/LIBRO RESUMENES_api_2013.pdf

9.- Corredoira-Sánchez J, García-Garrote F, Rabuñal R, López-Roses L, García-País M J, Castro E, et al. Association between bacteremia due to Streptococcus gallolyticus subsp. gallolyticus (Streptococcus bovis I) and colorectal neoplasia: a cse-control study. Clin Infect Dis 2012; 55: 491-6.

10.- Corredoira J, Alonso M P, García-Garrote F, García-Pais M J, Coira A, Rabuñal R, et al. Streptococcus bovis group and biliary tract infections: an analysis of 51 cases. Clin Microbiol Infect 2014; 20: 405-9.

11.- Sheng W H, Chuang Y C, Teng L J, Hsueh P R. Bacteraemia due to Streptococcus gallolyticus subsp pasteurianus is associated with digestive tract malignancies and resistance to macrolides and clindamycin. J Infect 2014; 69: 145-53. 